\title{
INFLUÊNCIA DO TIPO E CONCENTRAÇÃO DO SAL NAS PROPRIEDADES CORROSIVAS DOS FLUIDOS DE COMPLETAÇÃO*
}

\author{
Ycaro Jorge Maia da Costa ${ }^{1}$ \\ Guilherme Maciel Barbosa ${ }^{2}$ \\ Keila Regina Santana Fagundes ${ }^{3}$ \\ Rodrigo Cesar Santiago ${ }^{4}$ \\ Jardel Dantas da Cunha ${ }^{5}$ \\ Gecílio Pereira da Silva ${ }^{6}$
}

\section{Resumo}

Durante o processo para colocar um poço petrolífero em funcionamento existem algumas etapas a serem seguidas. Várias fases devem ser cumpridas para que 0 óleo bruto armazenado seja extraído. Dentre essas, duas são muito importantes: a perfuração, que é a fase de construção do poço em si, e logo após essa à de completação, a qual tem como objetivo deixar o poço em condições de produção. Os fluidos de completação extremamente salinos foram preparados empregando os seguintes sais: cloreto de sódio $(\mathrm{NaCl})$, cloreto de potássio $(\mathrm{KCl})$ e Cloreto de cálcio $\left(\mathrm{CaCl}_{2}\right)$ nas concentrações de 50, 100 e $150 \mathrm{lb} / \mathrm{bbl}$. O tipo do sal desencadeia uma seria de efeitos relacionados a taxa corrosiva e morfologia do ataque corrosivo, onde o diagrama de Pareto foram considerados estatisticamente significante ao nível de 95\% de confiança com significância quadrática (Q) dos parâmetros e das interações entre os mesmos. Observou-se que os mínimos e máximos valores da taxa de corrosão ocorrem quando os fluidos de completação foram confeccionados com $\mathrm{NaCl}$ e $\mathrm{CaCl}_{2}$.

Palavras-chave: Completação; Revestimentos; Fluido de Completação, Corrosão.

\section{INFLUENCE OF SALT TYPE AND CONCENTRATION ON CORROSIVE PROPERTIES OF COMPLETING FLUIDS}

\begin{abstract}
Throughout the process of putting a working oil well there are a few steps to follow. Several stages must be fulfilled in order for the stored crude oil to be extracted. Among these phases, two are very important: drilling, which is the phase of construction of the well itself, and soon after this begins the completion, which aims to leave the well in production conditions. The extremely saline completion fluids were prepared using the following salts: sodium chloride $(\mathrm{NaCl})$, potassium chloride $(\mathrm{KCl})$ and calcium chloride $\left(\mathrm{CaCl}_{2}\right)$ at the concentrations of 50,100 and $150 \mathrm{lb} / \mathrm{bbl}$. The salt type triggers a series of effects related to corrosive rate and corrosive attack morphology, where the Pareto diagram was considered statistically significant at the 95\% confidence level with quadratic significance (Q) of the parameters and their interactions. It was observed that the minimum and maximum values of the corrosion rate occur when the completion fluids were made with $\mathrm{NaCl}$ and $\mathrm{CaCl}_{2}$..
\end{abstract}

Keywords: Completing; Coating; Completing Fluid; Corrosion .

Graduando em Engenharia Mecânica, UFERSA, Mossoró, RN, Brasil.

Bacharel em Engenharia de Petróleo, UFERSA, Mossoró, RN, Brasil.

Doutora em Ciência e Engenharia do Petróleo, UFERSA, Mossoró, RN, Brasil

Doutor em Ciências e Engenharia de Materiais, UFERSA, Mossoró, RN, Brasil.

Doutor em Ciência e Engenharia do Petróleo, UFERSA, Mossoró, RN, Brasil.

Doutor em Química., UFERSA, Mossoró, RN, Brasil. 


\section{INTRODUÇÃO}

Durante o processo para colocar um poço de petróleo em funcionamento existem algumas etapas a serem seguidas entre a exploração e a explotação. Entre as etapas mais importantes a etapa de perfuração é a fase de construção do poço até chegar a zona de interesse e logo depois se dá a fase de completação, a qual tem a finalidade de deixar o poço estável e em condições de produzir em segurança e sem prejudicar o reservatório [1].

Conhecimento já existente da formação e da reologia dos campos produtores no estado do Rio Grande do Norte a escolha dos fluidos, empregados tanto na etapa de perfuração quanto os de completação variam muito de poço para poço. O fluido de completação geralmente é uma solução salina, onde características como: custo, pressão e temperatura da formação são necessárias afim de evitar-se danos à formação.

A corrosão é freqüentemente encontrada na indústria de petróleo e gás natural e ocorre em todos os estágios de produção, desde a prospecção até às instalações de processamento [2]. O processo corrosivo nos revestimentos ocasiona entre outros problemas custos elevado de reparo, perda de produção, problemas ambientais e até mesmo ameaçar a segurança pessoal [3, 4, 5, 6].

A camada passiva formada tem papel fundamental no mecanismo, na cinética e na morfologia de corrosão onde a mesma se torna o fator de controle da taxa de corrosão, antes da evolução catódica do hidrogênio [7, 8].

Diante do exposto, o presente trabalho tem como objetivo analisar o potencial de corrosividade dos sais cloreto de sódio $(\mathrm{NaCl})$, cloreto de potássio $(\mathrm{KCl})$ e Cloreto de cálcio $\left(\mathrm{CaCl}_{2}\right)$ nas concentrações de 50, 100 e $\left.150 \mathrm{lb} / \mathrm{bbl}\right)$, o que torna uma referência à seleção de fluidos de Completação.

\section{MATERIAIS E MÉTODOS}

Os fluidos de completação foram preparados empregando os sais: cloreto de sódio $(\mathrm{NaCl})$, cloreto de potássio $(\mathrm{KCl})$ e Cloreto de cálcio $\left(\mathrm{CaCl}_{2}\right)$ nas concentrações de 50, 100 e $150 \mathrm{lb} / \mathrm{bbl}$. Os fluidos foram agitados e medidas as propriedades de salinidade e densidade.

Os testes foram procedidos em célula de três eletrodos: o eletrodo de trabalho foi confeccionado a partir do aço API 5L A210 com composição: Fe, 98.88; C, 0.20; $\mathrm{Mn}, 0.91 ; \mathrm{P}, 0.01 ; \mathrm{S}, 0.006 ; \mathrm{Ni}, 0.04 ; \mathrm{Cr}, 0.05, \mathrm{Mo}, 0.009 ; \mathrm{Cu}, 0.04$ e área de 1,2568 $\mathrm{cm}^{2}$. O contra eletrodo empregado foi de platina e o eletrodo de referencia de Prata/Cloreto de Prata $(\mathrm{Ag} / \mathrm{AgCl})$. As medidas foram feitas em cima de um potencial de circuito aberto em uma faixa de potencial de $-300 \mathrm{mV}$ a $+300 \mathrm{mV}$ e com uma varredura de $0,001 \mathrm{~V} / \mathrm{s}$.

Para verificarmos se os parâmetros estudados apresentam influencia no potencial de corrosividade dos fluidos de completação, foi realizada uma analise dos parâmetros: tipo do sal e concentração do sal. O desenvolvimento da analise da combinação entre os parâmetros foi empregado o planejamento fatorial com dois níveis e três variáveis resultando em nove experimentos as quais foram analisadas por diagrama de Pareto e superfície de resposta. Os valores mínimos, médios e máximos foram delimitados para cada parâmetro (Tabela 1). 
Tabela 1. Níveis dos parâmetros analisados

\begin{tabular}{lccc}
\hline Parâmetro & $(-1)$ & $\begin{array}{c}\text { Níveis } \\
(0)\end{array}$ & $(+1)$ \\
\hline Tipo do sal & $\mathrm{NaCl}$ & $\mathrm{CaCl} 2$ & $\mathrm{KCl}$ \\
\hline Concentração do sal & $50 \mathrm{lb} / \mathrm{bbl}$ & $150 \mathrm{lb} / \mathrm{bbl}$ & $150 \mathrm{lb} / \mathrm{bbl}$ \\
\hline
\end{tabular}

\section{RESULTADOS E DISCUSSÃO}

\subsection{Salinidade e densidade}

A Tabela 2 apresenta as propriedades de densidade e salinidade para os fluidos confeccionados com os sais $\mathrm{KCl}, \mathrm{NaCl}$ e $\mathrm{CaCl}_{2}$

Tabela 2. Propriedades de densidade e salinidade para os fluidos confeccionados com os sais $\mathrm{KCl}$, $\mathrm{NaCl}$ e $\mathrm{CaCl}_{2}$.

\begin{tabular}{ccccccc}
\hline & \multicolumn{3}{c}{ Densidade (lb/gal) } & \multicolumn{3}{c}{ Salinidade } \\
\hline $\mathrm{Sal}$ & 50 & 100 & 150 & 50 & 100 & 150 \\
\hline $\mathrm{KCl}$ & 9,1 & 9,7 & 9,9 & 6,2 & 11,25 & 13,15 \\
$\mathrm{NaCl}$ & 9,2 & 9,9 & 10,2 & 7,6 & 14,35 & 17,15 \\
$\mathrm{CaCl}_{2}$ & 9,15 & 9,7 & 10,2 & 6,5 & 12,0 & 16,21 \\
\hline
\end{tabular}

O coeficiente de Solubilidade representa a maior massa que pode ser dissolvida em certa quantidade padrão de um solvente, em determinada temperatura. De acordo com a literatura, $\mathrm{NaCl}, \mathrm{KCl}$ e $0 \mathrm{CaCl}_{2}$ apresentam, respectivamente, $35,7 \mathrm{~g} / 100 \mathrm{~mL}, 78,6 \mathrm{~g} / 100 \mathrm{~mL}$ e $330 \mathrm{~g} / 100 \mathrm{~mL}$ de $\mathrm{H}_{2} \mathrm{O}$, respectivamente. Por outro lado, com base nos dados apresentados na Figura 1, as densidades de 9,9, 10,2 e 10,2 foram as máximas encontradas pela adição de $\mathrm{NaCl}$, $\mathrm{KCl}$ e $\mathrm{CaCl}_{2}$, respectivamente, nas concentrações utilizadas.

Essa discrepância com os dados obtidos na literatura é atribuída a pureza de cada sal utilizado e consequentemente, a massa molar de cada um dos compostos. Esses valores encontram-se abaixo do ponto de saturação de sal nos fluidos, conforme pode ser visualizado na Figura 2, onde pode-se observar ainda uma tendência ao incremento de densidade. No entanto, em virtude das concentrações atribuídas para efeito de estudo estatístico, foram utilizados os níveis: mínimo (50 $\mathrm{lb} /$ gal), central (100 lb/gal) e máximo (150 lb/gal) das concentrações.

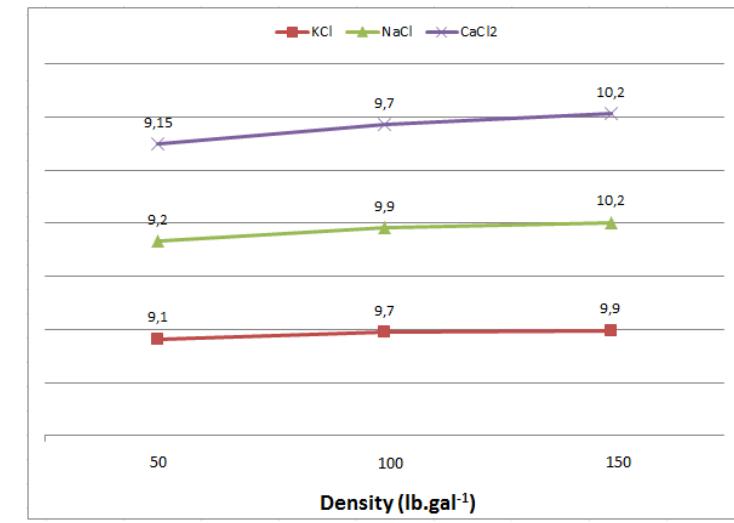

a)

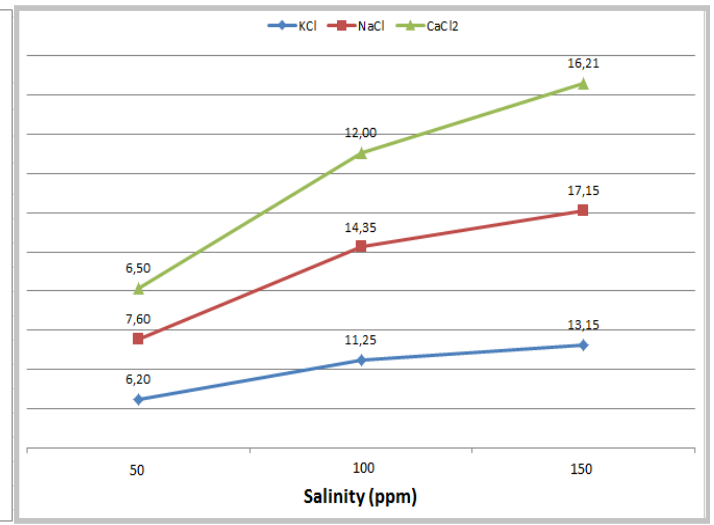

b)

Figura 1. a) Densidade dos fluidos em função da concentração do sal e b) Salinidade dos fluidos em função da concentração do sal. 


\subsection{Corrosividade dos fluidos de completação}

A figura 2 apresenta a superfície de resposta e diagrama de Pareto, do efeito concentração do sal versus o tipo de sal, tendo como resposta a taxa de corrosão. No diagrama para os parâmetros analisados tendo a taxa de corrosão como variável resposta. Os valores negativos apresentados no diagrama de Pareto (Figura 2.b) indica que tal variável contribui no sentido de diminuir o valor esperado para a resposta analisada, que neste caso e a taxa corrosiva caso ocorra a mudança do nível mínimo ao máximo para a variável estudada. Os resultados são considerados estatisticamente significante ao nível de $95 \%$ de confiança com significância quadrática $(Q)$ dos parâmetros e das interações entre os mesmos. Neste caso o tipo de sal desencadeia uma seria de efeitos relacionados a taxa de corrosão e morfologia do ataque corrosivo figuras 03, 04 e 05.

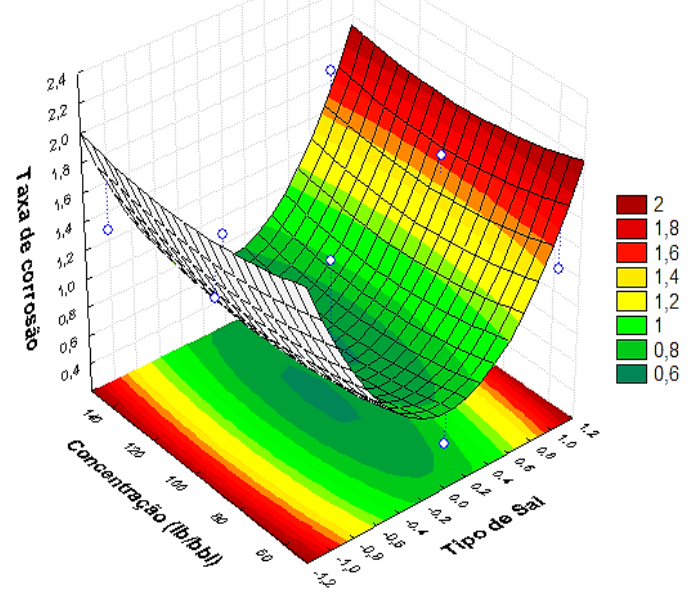

a)

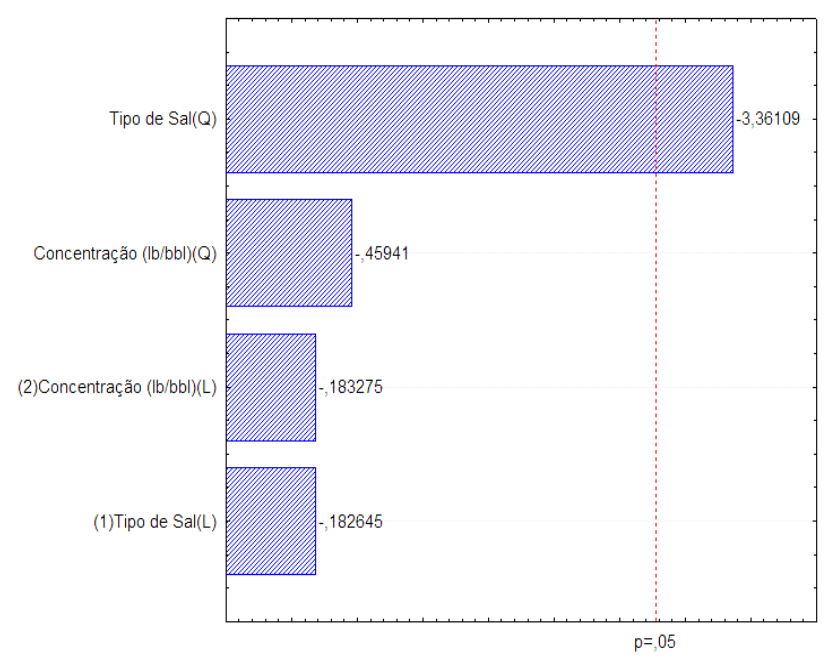

b)

Figura 2. a) Superfície de resposta para os parâmetros analisados concentração do sal e tipo do sal e b) Diagrama de Pareto para a concentração do sal e tipo do sal.

Foi observado que os máximos valores da taxa de corrosão ocorrem quando o fluido de completação é confeccionado com o $\mathrm{NaCl}(-1)$ e neste caso quando baixas concentrações deste sal $(50 \mathrm{lb} / \mathrm{bbl})$ são empregadas. Em outro extremo foi observado que o sal $\mathrm{KCl}(+1)$ apresenta os máximos valores de taxa de corrosão quando o fluido é elaborado com concentrações de $150 \mathrm{lb} / \mathrm{bbl}$. Os fluidos de completação confeccionados com $\mathrm{CaCl}_{2}$ (0) apresentaram comportamento intermediário no que remete-se a taxa de corrosão, onde observa-se comportamento semelhante ao sal $\mathrm{KCl}(+1)$. As variações das taxas corrosivas podem ser explicadas em virtude do caratê do sal quando solubilizado.

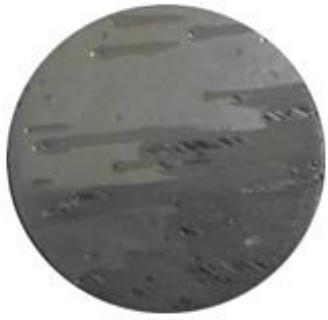

(a)

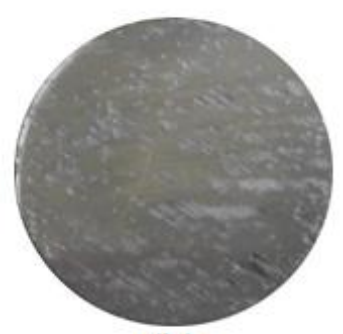

(b)

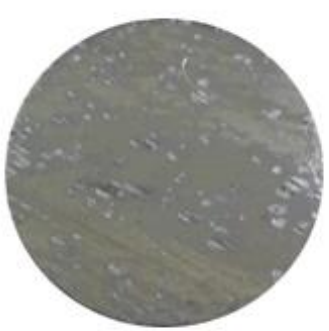

(c) 
Figura 3. Analise da superfície após ensaio de polarização linear em fluidos de completação preparados com $\mathrm{CaCl}_{2}$ nas concentrações: a) 50 ppm, b) 100 ppm e c) 150 ppm.

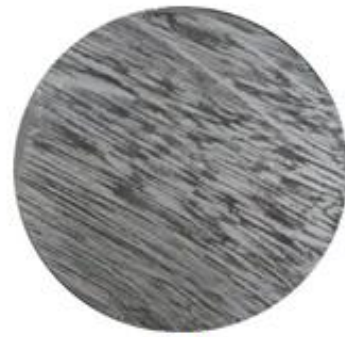

(a)

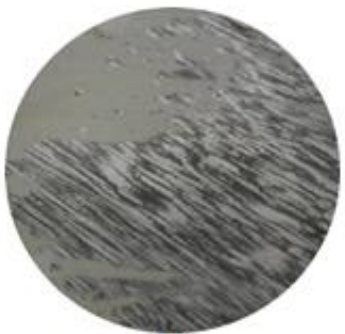

(b)

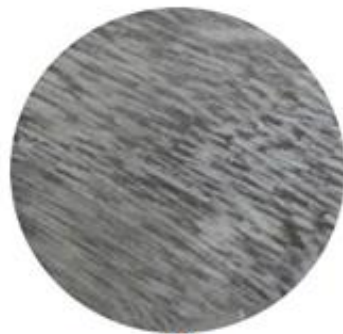

(c)

Figura 4. Analise da superfície após ensaio de polarização linear em fluidos de completação preparados com KCl nas concentrações: a) 50 ppm, b) 100 ppm e c) 150 ppm.

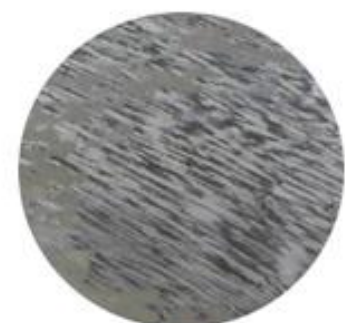

(a)

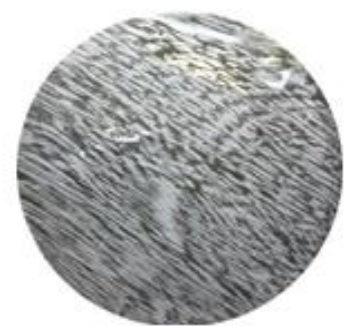

(b)

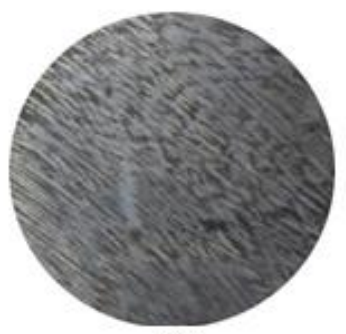

(c)

Figura 5. Analise da superfície após ensaio de polarização linear em fluidos de completação preparados com $\mathrm{NaCl}$ nas concentrações: a) 50 ppm, b) 100 ppm e c) 150 ppm.

Nas figuras 4 e 5, não observa-se a preferencia pela ocorrência do processo corrosivo localizado. Na Figura 3 observa-se a presença de processo corrosivo localizado com pites com profundidade média dos pites com 22 microns.

As figuras 6, 7 e 8, apresentam o comportamento das curvas de polarização potenciodinâmicas para os sais estudados nas concentrações de 50, 100 e 150 $\mathrm{lb} / \mathrm{bbl}$, respectivamente.

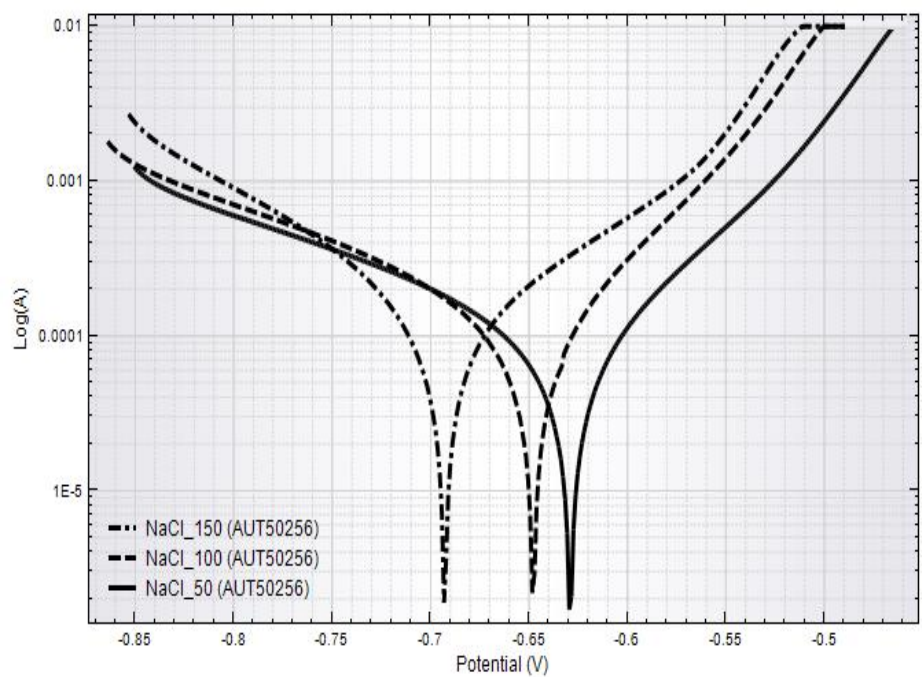

Figura 6. Curvas de polarização potenciodinâmicas para os fluidos confeccionados com o $\mathrm{NaCl}$ nas concentrações 50,100 e $150 \mathrm{lb} / \mathrm{bbl}$. 
A figura 6 apresenta para o fluido confeccionado com o $\mathrm{NaCl}$ com concentração de $50 \mathrm{lb} / \mathrm{bbl}$ um potencial de corrosão mais elevado que os demais fluidos confeccionados com concentração de 100 e $150 \mathrm{lb} / \mathrm{bbl}$, onde observou-se uma redução na taxa corrosiva em $59 \%$ quando comparados os fluidos nas concentrações de 50 e $150 \mathrm{lb} / \mathrm{bbl}$. As curvas apresentaram comportamentos distintos para os ramos anódicos das curvas de Tafel onde o aumento da concentração promoveu uma mudança na inclinação da curva que indicam uma passivação da superfície reduzindo assim, a corrente da corrosão. Os ramos catódicos indicaram comportamentos semelhantes com pouca mudança na liberação de hidrogênio.

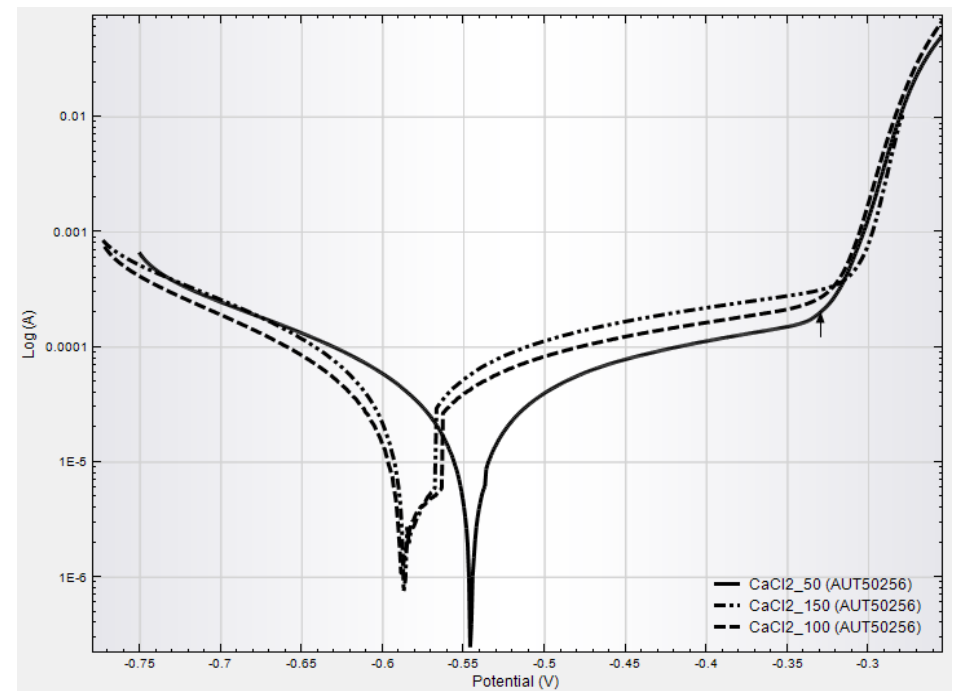

Figura 7. Curvas de polarização potenciodinâmicas para os fluidos confeccionados com $\circ \mathrm{CaCl}_{2}$ nas concentrações 50, 100 e $150 \mathrm{lb} / \mathrm{bbl}$.

$\mathrm{Na}$ figura 7, apresentou dois comportamentos distintos para os fluidos confeccionados com o $\mathrm{CaCl}_{2}$ nas concentrações de 100 e $150 \mathrm{lb} / \mathrm{bbl}$. No fluido confeccionado com concentração de 100 e $150 \mathrm{lb} / \mathrm{bbl}$ observa-se uma alteração no potencial com uma súbita elevação em potenciais de -0,586V, sendo este atribuído a formação de uma camada passiva intermediária, que a medida que o potencial é elevado a mesma mantém-se estável. Em potenciais acima de - $0,32 \mathrm{~V}$ um aumento abrupto do potencial indica possivelmente a ruptura da camada formada. 


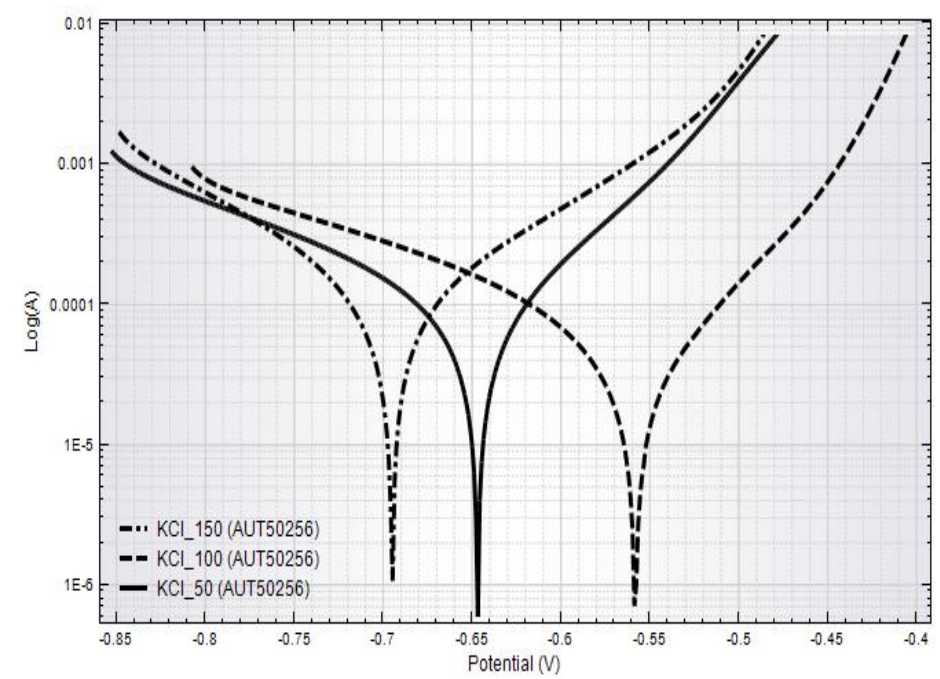

Figura 8. Curvas de polarização potenciodinâmicas para os fluidos confeccionados com o $\mathrm{KCl}$ nas concentrações 50, 100 e $150 \mathrm{lb} / \mathrm{bbl}$.

As curvas de polarização para os fluidos confeccionados com o $\mathrm{KCl}$, figura 8 , apresentaram comportamentos distintos a medida que a concentração do sal é elevada, onde foi observado um aumento em $79 \%$ na taxa corrosiva quando comparado os fluidos nas concentrações de 50 e $150 \mathrm{lb} / \mathrm{bbl}$.

\section{CONCLUSÃo}

A seleção dos fluidos de completação no que se remete ao tipo de sal a escolha do Cloreto de cálcio e do cloreto de potássio foram as escolhas com resultados mais significativos no que se remete ao tipo do processo corrosivo que em todos as concentrações estudadas foi o processo uniforme. A cerca do uso do cloreto de cálcio a escolha do mesmo se tornaria inviável dado que em todos os casos foi observado a preferencia pelo processo corrosivo localizado que em termos de futuras intervenções implicaria em operações de cimentação secundaria (squeezer) nos revestimentos.

Quanto a concentração do sal de acordo com os resultados apresentados observou-se que as densidades de 9,9, 10,2 e 10,2 foram as máximas encontradas pela adição de $\mathrm{NaCl}, \mathrm{KCl}$ e $\mathrm{CaCl}_{2}$, respectivamente.

Foi observado que os máximos valores da taxa de corrosão ocorrem quando o fluido de completação são confeccionado com o NaCl e em baixas concentrações deste sal $(50 \mathrm{lb} / \mathrm{bbl})$. Os fluidos de completação confeccionados com $\mathrm{CaCl}_{2}$ apresentaram comportamento com menores taxas de corrosão, mas ocorre preferencia pelo processo corrosivo localizado.

\section{REFERÊNCIAS}

[1] Zhu Kuanliang, Feng Jinghai, Han Fei, Li Nan, Pilot test of horizontal drilling and completion techniques in Nanpu Oilfield, PETROL. EXPLOR. DEVELOP., 2009, 36 (5): 658-663. 
[2] LI, J.; WANG, C.; LIU, J.; LIU, L.; YIN, Z.; ZHANG, Y.; LI, H.; HE, F. "Corrosion analysis, and use of an inhibitor in oil wells". Research Chemistry Intermed. v. 40, p. 649-660, 2014.

[3] MACK, R.,WILLIAMS, C., LESTER, S., Stress Corrosion Cracking of a Cold Worked 22Cr Duplex Stainless Steel Production Tubing in a High Density Clear Brine CaC12 Packer Fluid - Results of the Failure Analysis at Deep Alex and Associated Laboratory Experiments. Corrosion/2002, Houston TX: NACE Paper No. 02067:1-3.

[4] MOURA, Jéssyca Souza et al. Corrosão: colunas de perfuração de poços de petróleo. Cadernos de Graduação, Maceió, v. 3, n. 1, p.65-74, nov. 2015.

[5] S.D. Zhu, J.F. Wei, Z.Q. Bai, G.S. Zhou, R. Cai, Failure analysis of P110 tubing stringin the ultra-deep oil well, Eng. Fail. Anal. 18 (2011) 950-962.

[6] S.D. Zhu, J.F. Wei, R. Cai, Z.Q. Bai, G.S. Zhou, Corrosion failure analysis of high strength grade super 13Cr-110 tubing string, Eng. Fail. Anal. 18 (2011)2222-2231.

[7] Kinsella, Y. J.; Tan, Y. J.; Bailey, S. "Electrochemical Impedance Spectroscopy and Surface Characterization Techniques to Study Carbon Dioxide Corrosion Product Scales", Corrosion, v. 54, n. 10, p.835-842, 1998.

[8] Dezhi Zeng, Rui Chen, Zhi Zhang, Liyun Shao, Guoping Li, Gang Tian, Taihe Shi. Research on Stress Corrosion Sensitivity of C110 Casing in Wellbore Protection Fluid. Energy Procedia, 2012,16 (B):816-821 